\title{
Skyrmions in the Fractional Quantum Hall Effect
}

\author{
R.K. Kamilla, X.G. Wu, and J.K. Jain \\ Department of Physics, State University of New York at Stony Brook, Stony Brook, New York \\ $11794-3800$
}

(Feb. 16, 1996)

\begin{abstract}
It is verified that, at small Zeeman energies, the charged excitations in the vicinity of $1 / 3$ filled Landau level are skyrmions of composite fermions, analogous to the skyrmions of electrons near filling factor unity. These are found to be relevant, however, only at very low magnetic fields.
\end{abstract}

PACS numbers: $73.40 . \mathrm{Hm}$

Typeset using REVTEX

${ }^{0}$ Accepted for publication in Solid State Communications (1996). 


\section{A. Introduction}

Since the discovery of the fractional quantum Hall effect (FQHE) [1], the nature of various states of interacting electrons confined to their lowest LL has been a subject of great interest. Recently, a new theory is being explored according to which the system of strongly correlated electrons at magnetic field $B$ maps on to a system of weakly interacting composite fermions at an effective magnetic field $B^{*}=B-2 m \rho \phi_{0}$, where $m$ is an integer, $\rho$ is the electron density, and $\phi_{0}=h c / e$ is the quantum of flux [2]. The wave functions for the composite fermions are constructed by multiplying the wave functions of weakly interacting electrons at $B^{*}$ by the Jastrow factor $\prod_{j<k}\left(z_{j}-z_{k}\right)^{2 m}$, where $z_{j}=x_{j}+i y_{j}$ denotes the position of the $j$ th electron [2]. This simple theory successfully provides a detailed and unified description of various liquid states of electrons in the lowest Landau level (LL), in particular, of spinpolarized incompressible FQHE states and their charged and neutral excitations [2, 3], the compressible state at and near 1/2 filled LL [4], and incompressible odd-denominator FQHE states at small Zeeman energies [0, 6]. The last case is of relevance since the Zeeman energy is quite small in GaAs, and there exists good experimental evidence that many FQHE states in low-density samples are not fully polarized [5, 7]. The ground state at 1/3 is, however, fully polarized even in the absence of Zeeman splitting, and is well described by the Laughlin wave function [8], which is interpreted in the composite fermion (CF) scheme as one filled LL of composite fermions. Nakajima and Aoki [9] have shown that the (neutral) spin-wave excitation at filling factor $\nu=1 / 3$ can be quantitatively understood as the spinwave excitation of composite fermions at CF filling factor $\nu^{*}=1$. This Communication demonstrates analogous behavior for the (charged) skyrmion excitation near the $1 / 3$ filled LL at zero Zeeman energy. A 'hard-core' trial wave function is found to be rather accurate, and allows an estimation of the number of reversed spins in the CF-skyrmion as a function of the Zeeman energy.

It is convenient to use a spherical geometry [10, in which $N$ electrons move on the surface of a sphere under the influence of a radial magnetic field. For $N_{\phi}$ flux quanta threading the 
surface of the sphere, the degeneracy of the lowest LL is $2\left(N_{\phi}+1\right)$, where the factor of 2 originates from the electron spin. According to the composite fermion theory, interacting electrons at flux $N_{\phi}$ resemble weakly interacting composite fermions at flux

$$
N_{\phi}^{*}=N_{\phi}-2(N-1)
$$

The $1 / 3$ state occurs at $N_{\phi}=3(N-1)$, which, relates to composite fermions at $N_{\phi}^{*}=N-1$, corresponding to a CF filling factor $\nu^{*}=1$.

The quasiparticle (quasihole) of the $\nu=1$ or state is obtained by decreasing (increasing) the flux $N_{\phi}$ by one unit. At large Zeeman energies, the quasiparticle (QP) involves reversal of a single spin, and the quasihole $(\mathrm{QH})$ state is fully polarized. However, the spin configuration at small Zeeman energies is rather unusual, involving a reversal of possibly a large number of spins relative to the $\nu=1$ state [11]. Such a quasiparticle or quasihole is generically called a (QP- or QH-) skyrmion [12,13].

\section{B. $\nu=1$ skyrmion}

Let us first set the Zeeman energy equal to zero. Then, the total angular momentum $L$ and the total spin $S$ are good quantum numbers.

At $\nu=1\left(N_{\phi}=N-1\right)$, the ground state is fully polarized, as one might have anticipated from Hund's first rule. However, the Hund's rule is maximally violated just one flux quantum away, at $N_{\phi}=N$ (or $N_{\phi}=N-2$, which is related to $N_{\phi}=N$ by an exact particle-hole symmetry in the lowest LL); here the ground state has total spin $S=0$ and the total orbital angular momentum $L=0$ [1]. In fact, the spectrum here contains a low energy

branch of states with quantum numbers $(L, S)=(0,0),(1,1), \ldots\left(\frac{N}{2}, \frac{N}{2}\right)$ (with $N$ taken to be an even integer for convenience), satisfying the property $L=S$ [14 [16]. The existence of this 'skyrmion' branch can be understood as follows. Consider a hard-core delta function interaction. The states in which electrons completely avoid one another irrespective of their spin will have zero interaction energy, and will be referred to as 'hard-core' states. 
It has been known from numerical diagonalization [11, 14], and also proven analytically [16], that for $N_{\phi}=N$, the hard-core states occur at precisely the quantum numbers given above. Further, it has also been confirmed numerically that the low-energy eigenstates of the Coulomb interaction are indeed almost identical to the hard-core states [14] (although the longer range part of the Coulomb interaction removes their degeneracy).

The important question then is: What is the energy ordering of these states for the Coulomb interaction? The Hund's rule answers this question successfully, provided it is applied to composite fermions rather than electrons. Repeated applications of the CF transformation, which progressively zooms into the lower energy states, shows that the energy increases with $S$, consistent with exact diagonalization results [14].

The hard-core wave function of the $\nu=1$ skyrmion is determined completely by symmetry (i.e., is independent of the strength of the delta function interaction), since there is only one hard-core state for any given set of $L, S, L_{z}$ and $S_{z}$ quantum numbers. We proceed to study large systems using the the hard-core wave function for the QH-skyrmion proposed by MacDonald, Fertig, and Brey [16]. For $R$ reversed spins (relative to the fully polarized quasihole), this wave function is given by

$$
\Psi_{S K}(R)=\left[\sum_{i_{1}, \ldots, i_{R}}\left(z_{j_{1}} \ldots z_{j_{N-R}}\right)\left(\downarrow_{i_{1}} \ldots \downarrow_{i_{R}} \uparrow_{j_{1}} \ldots \uparrow_{j_{N-R}}\right)\right] \Phi_{1}
$$

where $\uparrow$ and $\downarrow$ denote up and down spins, $j$ 's denote particles other than $i_{1}, \ldots, i_{R}$, and $\Phi_{1}$ is given by

$$
\Phi_{1}=\prod_{j<k}\left(z_{j}-z_{k}\right) \exp \left[-\frac{1}{4} \sum_{i}\left|z_{i}\right|^{2}\right] .
$$

$\Psi_{S K}$ has a well defined $S$ in the limit of large $N$ [16]. For the spherical geometry, the product $z_{j_{1} \ldots} \ldots z_{j_{N-R}}$ is replaced by $v_{j_{1}} \ldots v_{j_{N-R}} u_{i_{1}} \ldots u_{i_{R}}$, where $u$ and $v$ are spinor coordinates [10], and

$$
\Phi_{1}=\prod_{j<k}\left(u_{i} v_{j}-v_{i} u_{j}\right),
$$

which is the wave function of the lowest filled LL in the spherical geometry.

We have computed the Coulomb energy of $\Psi_{S K}(R)$ for several values of $N$ by Monte Carlo, shown in Fig. 1 [17. The results are reasonably close to the thermodynamic limit, 
especially for the energy differences between different $R$ (for small $R$ ). The energy of the 50 electron system is well approximated by (in units of $e^{2} / \epsilon l$ )

$$
E_{1}^{(0)}(R)=0.313+0.23 \exp \left(-0.25 R^{0.85}\right) .
$$

It approaches $0.313 e^{2} / \epsilon l$ in the limit of large $R$, in agreement with the result of Ref. [13].

At strictly zero Zeeman splitting, the skyrmion ground state is a spin singlet, i.e., it involves a spin-reversal for half of the electrons. However, this limit is academic, since, even for a very small Zeeman energy, the lowest energy state of the skyrmion has only a finite number of reversed spins, as anticipated theoretically by Fertig et al. [18] and confirmed in several recent experiments [19 21. In the presence of a finite Zeeman energy, a term $g^{*} \mu_{B} B R$ [22] must be added to $E^{(0)}(R)$ to get the full energy $E(R)$. For parameters appropriate to GaAs (Lande- $g$ factor $g^{*}=0.44$, effective electron mass $m^{*}=0.067 m_{e}$ ), and converting all energies into Kelvin $\left(g^{*} \mu_{B} B \approx 0.30 B[T] K, e^{2} / \epsilon l \approx 50 \sqrt{B[T]} K, \hbar \omega_{c} \approx 20 B[T] K, B[T]\right.$ measured in Tesla), the total energy of the skyrmion is given by

$$
E_{1}(R)=\left[15.7+11.5 \exp \left(-0.25 R^{0.85}\right)\right] \sqrt{B}+0.30 B R .
$$

The magnetic field above which the minimum occurs at $R=0$ (i.e., the quasihole is fully polarized), estimated by the condition $E(0)=E(1)$, is $B \approx 72 T$. In general, the number of reversed spins is approximately given by the equation

$$
0.15 \ln (R)+0.25 R^{0.85}=\ln (8.1 / \sqrt{B}) .
$$

To take some typical values, for $B=2 T$ and $7 T$ the skyrmion is found to have $R \approx 7.8$ and 4.5 reversed spins, respectively, in good agreement with previous theoretical [18] and experimental [19 21] results. Note also that spin-reversal produces a substantial correction to the $R=0 \mathrm{QH}$ energy; already for $R=8$, the energy is quite close to $E_{1}(R=\infty)$.

\section{Skyrmions near $\nu=1 / 3$ and $\nu=1 / 5$}

Now we come to the skyrmion excitations near the $\nu=1 / 3$ state. We first set the Zeeman splitting to zero. At precisely $\nu=1 / 3$, the system is equivalent to $\nu^{*}=1$ of composite 
fermions, and is thus expected to be fully polarized, as found in numerical studies. The QH-skyrmion $N_{\phi}=3(N-1)+1$ maps into $N$ fermions at $N_{\phi}^{*}=N$, and the QP-skyrmion $N_{\phi}=3(N-1)-1$ maps into $N$ fermions at $N_{\phi}^{*}=N-2$.

We have numerically studied a system of six electrons at $N_{\phi}=14$ and 16 . The size of the Hilbert space can be reduced drastically by confining to the sector with $L_{z}=S_{z}=0$, but is still too large for exact diagonalization. (For $N_{\phi}=16$, the total number of states in this sector is 16,004 .) We have obtained several low-energy states by Lanczos technique. The CF analogy predicts that the low-energy states of the QP-skyrmion have quantum numbers $(L, S)=(0,0),(1,1)$, and $(2,2)$, and those of the QH-skyrmion occur at $(L, S)=$ $(0,0),(1,1),(2,2)$, and $(3,3)$. Our results confirm this: the lowest energy states with spins $S=0,1,2$ (and also 3 for $N_{\phi}=16$ ) indeed satisfy the property $L=S$, with energy increasing with $S$.

To further ensure the validity of the CF mapping, we construct CF wave functions for the skyrmion at each $(L, S)$. It was shown in Ref. [23] that at small Zeeman energies, the composite fermions (i.e., fermions at $N_{\phi}^{*}$ ) cannot be taken as non-interacting. We consider two types of interactions, Coulomb and hard-core, between them. The 'hard-core' ['Coulomb'] trial wave function for composite fermions is given by Jastrow factor ( $\Phi_{1}^{2}$ for the spherical geometry) times the eigenstate of the hard-core [Coulomb] interaction for the $\nu=1$ skyrmion. (Multiplication by $\Phi_{1}^{2}$ does not alter the $L$ and $S$ quantum numbers.) As shown in Tables I and II, the Coulomb energies of these states deviate from the exact Coulomb energy by $\sim 0.1 \%$ or less, establishing that the $\nu=1 / 3$ skyrmion of electrons is indeed the $\nu=1$ skyrmion of composite fermions.

We note that there is no exact symmetry relating the QP- and QH-skyrmions of the $\nu=1 / 3$ state. However, they are related by particle-hole symmetry when viewed in terms of composite fermions, clarifying the physics underlying the similarity between their low energy spectra.

For large $N$, we use $\Phi_{1}^{2} \Psi_{S K}(R)$ for the hard-core wave function for the 1/3-QH-skyrmion. Its energy is shown in Fig. 2 for several values of $N$. For $N=50$ : 


$$
E_{1 / 3}^{(0)}(R) /\left(e^{2} / \epsilon l\right)=0.069+0.024 \exp \left(-0.38 R^{0.72}\right)
$$

As before, adding the Zeeman term, expressing the energy in Kelvins and $B$ in Tesla, we get:

$$
E_{1 / 3}(R)=\left[3.45+1.20 \exp \left(-0.38 R^{0.72}\right)\right] \sqrt{B}+0.30 B R
$$

The magnetic field must be less than $\approx 1.6 T$ to produce any spin reversal. The number of reversed spins can be determined straightforwardly by minimizing $E_{1 / 3}(R)$.

The energy of the skyrmion near $\nu=1 / 5$ is estimated for GaAs to be (in K)

$$
E_{1 / 5}(R)=\left[1.7+0.37 \exp \left(-0.46 R^{0.75}\right)\right] \sqrt{B}+0.30 B R,
$$

from the wave function $\Phi_{1}^{4} \Psi_{S K}(R)$ for $N=40$ particles. For $B$ greater than $\approx 0.21 T$, the usual fully polarized quasihole has the lowest energy, and the skyrmion physics is not relevant.

These results show that the Zeeman energy is much more efficient in reducing the skyrmion size near $\nu=1 / 3$ and $1 / 5$. This is not surprising, since the inter-CF interaction at $\nu^{*}=1$ is much weaker than inter-electron interaction at $\nu=1$.

The energy of the $Q P$-skyrmion is harder to compute for large $N$. We only make two observations here. (i) The energies [17] of the QH and QP-skyrmions are equal at $\nu=1$. This may suggest that the energies of the two skyrmions should be equal also at $\nu=1 / 3$. This is not the case. (If it were, the gap to creating a QP-QH skyrmion pair at $\nu=1 / 3$, with zero Zeeman energy, would be $\sim 0.14 e^{2} / \epsilon l$, inconsistent with other estimates of $\sim 0.024 e^{2} / \epsilon l$ [13,24. For six electrons, the gap is $0.010 e^{2} / \epsilon l$.) The asymmetry can be understood as resulting from the fact that the vortices are attached only to electrons for both the QH- and QP-skyrmions. (ii) The range over which the QP-skyrmion energy extends, $E(0)-E(\infty)$, is estimated to be smaller than that for the QH-skyrmion 24]. (For $N=6$, it is $0.011 e^{2} / \epsilon l$ for the QP-skyrmion as opposed to $0.017 e^{2} / \epsilon l$ for the QH-skyrmion.) Therefore, any spin reversal on the QP side is expected to require even lower magnetic fields. 


\section{Conclusion}

A priori, one might expect skyrmions near all odd integer and the corresponding fractional filling factors. Experimental results of Schmeller et al. [20] show that the low-energy spectrum at $\nu=3,5, \ldots$ does not contain any skyrmion-like states, in accordance with the

theoretical expectation [14], also ruling out any skyrmion-like structure near $3 / 5,3 / 7,5 / 7$, etc. This leaves the possibility of skyrmions only near $\nu=1 /(2 m+1)$. While their observation is in principle possible at $\nu=1 / 3$ and $1 / 5$, it would require extremely low density GaAs samples.

Before closing, we note that we have not included above the effects of finite thickness and LL mixing, which are known to alter the QP and QH energies by as much as $50 \%$. We expect that these should lead to a more or less $R$ independent shift in the finite-size skyrmion energy, and therefore not change the above estimates significantly. This work was supported in part by the National Science Foundation under Grant No. DMR93-18739. 


\section{REFERENCES}

[1] D.C. Tsui, H.L. Stormer, and A.C. Gossard, Phys. Rev. Lett. 48, 1559 (1982).

[2] J.K. Jain, Phys. Rev. Lett. 63, 199 (1989); Phys. Rev. B 41, 7653 (1990); Science 266, 1199 (1994).

[3] G. Dev and J.K. Jain, Phys. Rev. Lett. 69, 2843 (1992); R.K. Kamilla, X.G. Wu, and J.K. Jain, Phys. Rev. Lett. 76, 1332 (1996); X.G. Wu and J.K. Jain, Phys. Rev. B 51, 1752 (1995); N. Bonesteel, Phys. Rev. B 51, 9917 (1995).

[4] B.I. Halperin, P.A. Lee, and N. Read, Phys. Rev. B 477312 (1993); R.R. Du et al., Phys. Rev. Lett. 70, 2944 (1993); W. Kang et al., ibid. 71, 3850 (1993); V.J. Goldman et al., ibid. 72, 2065 (1994); R.L. Willett et al., ibid. 71, 3846 (1993).

[5] R.R. Du, A.S. Yeh, H.L. Stormer, D.C. Tsui, L.N. Pfeiffer, and K.W. West, Phys. Rev. Lett. 75, 3926 (1995).

[6] X.G. Wu, G. Dev, and J.K. Jain, Phys. Rev. Lett. 71, 153 (1993).

[7] J.P. Eisenstein et al., Phys. Rev. Lett. 61, 997 (1987); ibid. 62, 1540 (1989); Phys. Rev. B 41, 7910 (1990); R.G. Clark et al., Phys. Rev. Lett. 62, 1536 (1989); L. W. Engel et al., Phys. Rev. B 45, 3418 (1992).

[8] R.B. Laughlin, Phys. Rev. Lett. 50, 1395 (1983).

[9] T. Nakajima and H. Aoki, Phys. Rev. Lett. 73, 3568 (1994).

[10] F.D.M. Haldane, Phys. Rev. Lett. 51, 605 (1983); T.T. Wu and C.N. Yang, Nucl. Phys. B 107, 365 (1976).

[11] E.H. Rezayi, Phys. Rev. B 43, 5944 (1991); ibid., 36, 5454 (1987).

[12] D.H. Lee and C.L. Kane, Phys. Rev. Lett. 64, 1313 (1990).

[13] S.L. Sondhi, A. Karlhede, S.A. Kivelson, and E.H. Rezayi, Phys Rev B 47, 16419 (1993). 
[14] J.K. Jain and X.G. Wu, Phys. Rev. B 49, 5085 (1994).

[15] S. He and X.C. Xie, Phys. Rev. B 53, 1046 (1996).

[16] A.H. MacDonald, H.A. Fertig, and L. Brey, SISSA preprint cond-mat/9510080.

[17] The energies quoted here are for the 'neutral' quasiparticle and quasihole, in the convention of A.H. MacDonald and S.M. Girvin, Phys. Rev. B 34, 5639 (1986).

[18] H.A. Fertig, L. Brey, R. Cote, and A.H. MacDonald, Phys. Rev. B 50, 11018 (1994).

[19] S.E. Barrett, R. Tycko, L.N. Pfeiffer, and K.W. West, Phys. Rev. Lett. 74, 5112 (1995).

[20] A. Schmeller, J.P. Eisenstein, L.N. Pfeiffer, and K.W. West, Phys. Rev. Lett. 75, 4290 (1995).

[21] E.H. Aifer, B.B. Goldberg, and D.A. Broido, Phys. Rev. Lett. 76, 680 (1996).

[22] The $B$ here is the total magnetic field, while the one that determines the magnetic length is the normal component. We will assume here that the two are the same. The analysis can be trivially generalized for tilted fields.

[23] X.G. Wu and J.K. Jain, Phys. Rev. B 49, 7515 (1994).

[24] K. Moon et al. Phys. Rev. B 51, 5138 (1995). 


\section{TABLES}

\begin{tabular}{|c|c|c|c|}
\hline \hline$(L, S)$ & exact Coulomb eigenstate & 'hard-core' trial state & 'Coulomb' trial state \\
\hline \hline$(0,0)$ & -0.4620 & -0.4617 & -0.4618 \\
\hline$(1,1)$ & -0.4616 & -0.4613 & -0.4613 \\
\hline$(2,2)$ & -0.4604 & -0.4600 & -0.4600 \\
\hline \hline
\end{tabular}

TABLE I. Energies of the exact Coulomb eigenstates and of the 'hard-core' and 'Coulomb' trial wave functions, explained in the text for the $\nu=1 / 3$-QP-skyrmion with $N=6$ electrons at $N_{\phi}=14$. The energies are given in units of $e^{2} / \epsilon l$, where $\epsilon$ is the background dielectric constant and $l=\sqrt{\hbar c / e B}$ is the magnetic length, and include the interaction with the positively charged neutralizing background.

\begin{tabular}{|c|c|c|c|}
\hline \hline \hline$(L, S)$ & exact Coulomb eigenstate & 'hard-core' trial state & 'Coulomb' trial state \\
\hline \hline$(0,0)$ & -0.4367 & -0.4362 & -0.4362 \\
\hline$(1,1)$ & -0.4364 & -0.4359 & -0.4360 \\
\hline$(2,2)$ & -0.4357 & -0.4354 & -0.4353 \\
\hline$(3,3)$ & -0.4337 & -0.4333 & -0.4333 \\
\hline \hline \hline
\end{tabular}

TABLE II. Same as in Table I for the QH-skyrmion with $N=6$ electrons at $N_{\phi}=16$. 


\section{FIGURES}

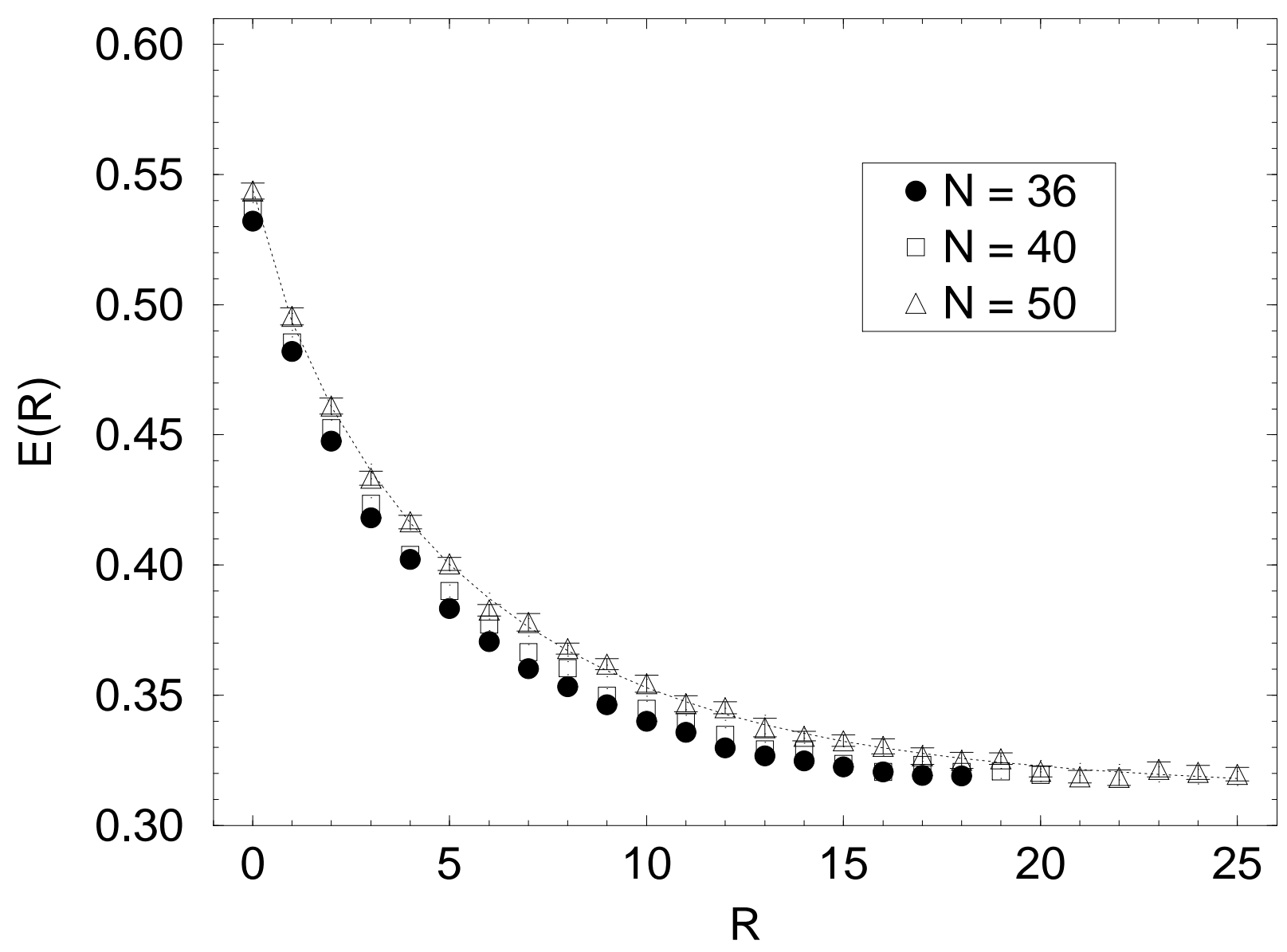

FIG. 1. Energy of the $\nu=1$ skyrmion in units of $e^{2} / \epsilon l$ for several systems. The energy is measured relative to the fully polarized $\nu=1$ state. The error bars are shown only for the $N=50$ electron system, and the dashed line plots Eq. (5). 


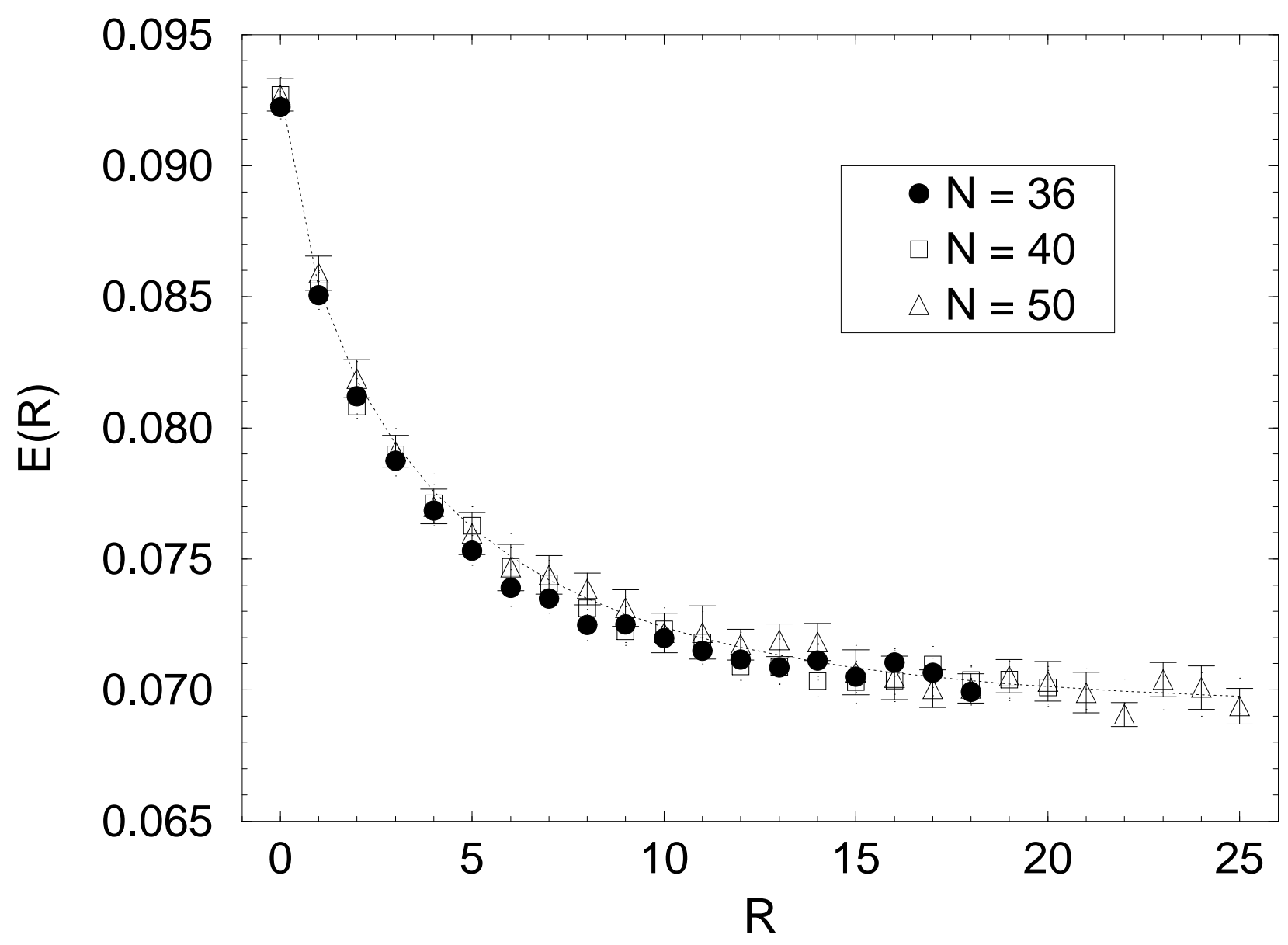

FIG. 2. Energy of the $\nu=1 / 3$ skyrmion measured relative to the fully polarized $1 / 3$ state. The error bars are shown only for the $N=50$ electron system, and the dashed line is a plot of Eq. (8). 